\title{
Evaluation of the rheological properties of photopolymers used in Polymer Jetting technology
}

\author{
Czesław Kundera ${ }^{1, *}$, and Tomasz Kozior ${ }^{1}$ \\ ${ }^{1}$ Kielce University of Technology, Faculty of Mechatronics and Mechanical Engineering, \\ al. Tysiaclecia Panstwa Polskiego 7, 25-314 Kielce, Poland
}

\begin{abstract}
The paper presents the results of tests related to relaxation of stresses in models of samples made using the technology of photo-curing of liquid polymer resins - polyjet modeling (PJM). The models of the samples were made using two types of materials: VeroWhite and FullCure 720. The tests were performed on samples in compliance with the PN-EN ISO 3384 standard. The technological parameter whose impact was analyzed was the orientation of the models on the virtual building platform, which was defined with three variability levels $\left(0^{\circ}, 45^{\circ}\right.$, and $\left.90^{\circ}\right)$. The tests demonstrated that the position of the models on the building platform has a significant impact on the relaxation of compressive stresses. Moreover, the most advantageous positions of the models on the building platform from the standpoint of relaxation of stresses were determined.
\end{abstract}

Keywords: Additive Technology, PJM, Stress Relaxation, Maxwell model, Mechanical Properties

\section{Introduction}

Rapid prototyping (RP) technologies have been known since over 30 years ago when some of the first technologies, such as Stereolithography (SLA) [14], Selective Laser Sintering (SLS) [15], and Fused Deposition Modelling (FDM) [16] were developed and patented. Due to the layer nature of construction, manufactured elements have a number of anisotropic properties that largely depend on the orientation of the models in relation to the building platform. This problem has been described in a number of publications related to both the mechanical properties of models and the accuracy of dimensions and shapes $[3,4,11,17]$. In the case of many additive technologies, such as SLS, PJM, and FDM, it is possible to build not only prototypes but also fully functional models, including the so-called overprints $[1,2$, 5, 9-13].

Additive technologies can be divided according to many criteria. One of the most important criteria is the form of the starting material, which can be a solid, e.g. a rod (FDM), a liquid (SLA, PJM), or a powder (SLS, SLM). Another very common criterion is the type

* Corresponding author: kundera@tu.kielce.pl

Reviewers: Ireneusz Malujda, Dominik Wilczyński 
of bonding between layers, such as gluing (LOM), sintering (SLS), and chemical reactions (SLA, PJM). Those technologies use such materials as both plastics and selected groups of metals.

The study described in this paper consisted in tests of relaxation of stresses in polymer samples made using the PJM technology from two materials (FullCure 720 and VeroWhite) in accordance with the PN-EN ISO 3384 standard [7]. Moreover, during the tests, the Statistica software and the Origin software was used to conduct a basic statistical analysis of the results of the tests and to approximate the relaxation curves using selected mathematical models.

\section{Research}

The stress relaxation tests were performed using the Inspekt Mini $3 \mathrm{kN}$ machine made by Hegewald \& Peschke [6]. The compression test was performed using two compression disks: a fixed upper disk and a swinging lower disk. The samples, designed in accordance with the standard [7], had the following dimensions: diameter $-13 \mathrm{~mm}$ and height $-6.3 \mathrm{~mm}$. The samples were made of two polymer resins: VeroWhite (1-9) and FullCure 720 (10-18). During the tests, the samples were subject to deformation equal to $10 \%$ of their height, i.e. $0.63 \mathrm{~mm}$. The set deformation was maintained for 90 minutes. After the test cycle was completed, relaxation of stresses was determined according to relationship 1 given in the PNEN ISO 3384 standard [7]; Table 1 shows the results of the measurements according to the orientation of the models on the building platform (Fig. 1 and 2).

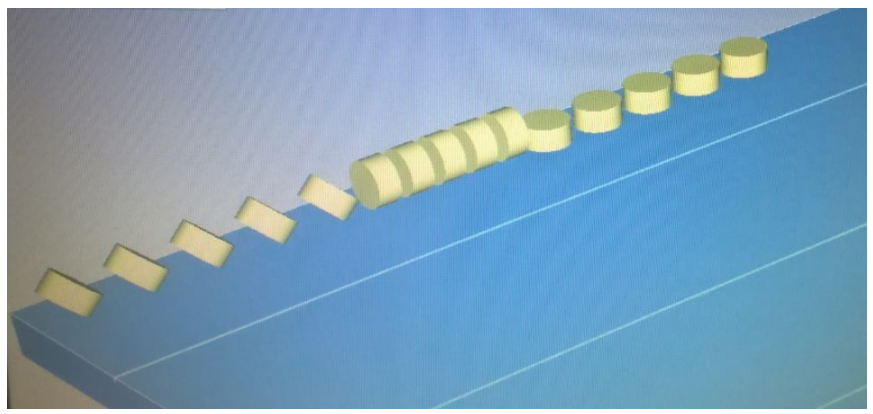

a)

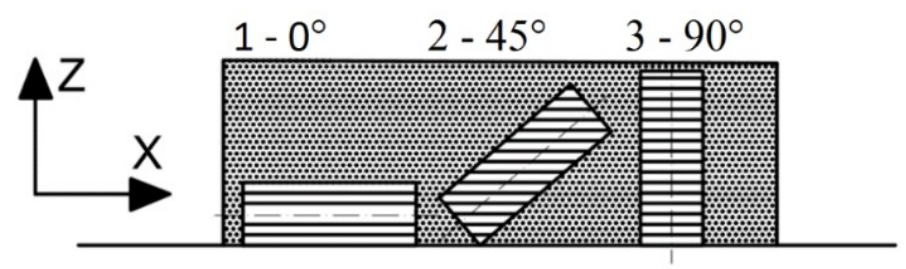

b)

Fig. 1. Orientation of the models on the building platform: a) Connex 350 machine, b) Cartesian coordinate system 


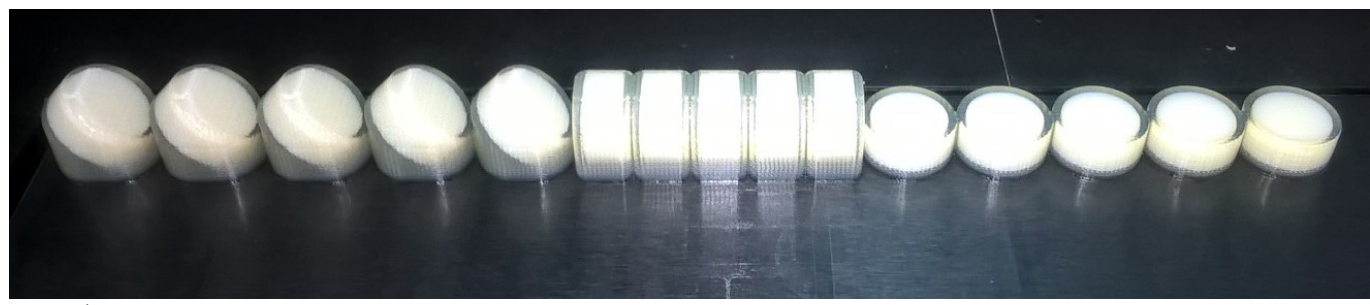

a)

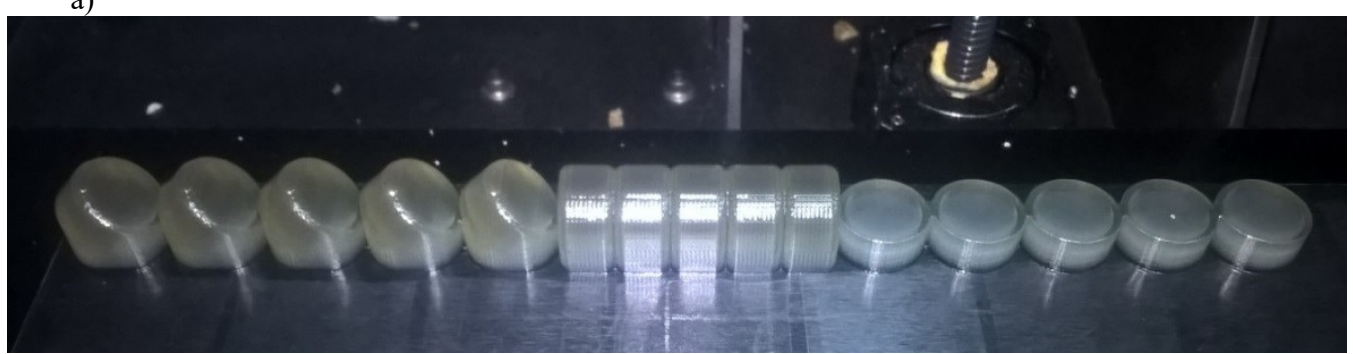

b)

Fig. 2. Samples: a) VeroWhite (numbers 1-9), b) FullCure 720 (numbers 10-18)

\section{Research results}

The tests concerning relaxation of compressive stresses were conducted in accordance with the ISO 3384 standard. The individual relaxation tests were conducted for 60 minutes, after stabilization for 30 minutes indicated with the symbol $R(t)_{30-90}$ and the total relaxation was determined after 90 minutes $R(t)_{c}$. The determined parameters of the rheological model that was used are shown in Tables 2 and 3. The stress relaxation value was calculated in accordance with relation 1 given in the aforementioned standard.

$$
R(t)=\frac{F_{0}-F_{t}}{F_{0}}
$$

where: $F_{0}-$ initial force $[\mathrm{N}], F_{T}-$ force after the time $t,[\mathrm{~N}]$.

Table 1 shows the results of the experimental measurements and the calculated indicators of relaxation of compressive stresses after 60 and 90 minutes. The figures that follow show the actual measurement charts for the samples made from the VeroWhite material (resin) (Fig. 3-5, samples 1-9) and the FullCure 720 material (Fig. 7-9, samples 10-18). The experimental relaxation curves were approximated using the exponential functions in the 2 and 3 form [8].

$$
\begin{gathered}
y=y_{0}+A_{1} e^{-x / t_{1}} \\
y=y_{0}+A_{1} e^{-x / t_{1}}+A_{2} e^{-x / t_{2}}
\end{gathered}
$$

where: $y$ - the stress function, $y_{0}, A_{1}, A_{2}, t_{1}, t_{2}$ - parameters of the stress functions.

Further in the description, functions (2) and (3) are referred to as relaxation functions. Approximations of the actual relaxation functions were performed in the Origin software using an implemented Levenberg-Marquardt algorithm. Figures 6 and 10 show examples of approximation with a five-parameter relaxation function (3) applied to the experimental results. Tables 2 and 3 show the parameter of the approximating functions (3) and the determined test indicators $\mathrm{R}^{2}$. 
Table 1. Research results

\begin{tabular}{|c|c|c|c|c|c|c|c|}
\hline Material & $\begin{array}{l}\text { Samples } \\
\text { number }\end{array}$ & $\mathbf{P d},{ }^{\circ}$ & $F_{\max }$ & $\mathbf{F}_{0}, \mathbf{N}$ & $F_{t}, N$ & $R(t)_{30-90}$ & $R(t)_{c}$ \\
\hline \multirow{15}{*}{ 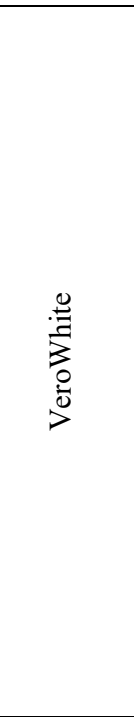 } & 1 & \multirow{5}{*}{$0^{\circ}$} & 1409.19 & 1364.94 & 1355.24 & 0.007 & 0.038 \\
\hline & 2 & & 1264.95 & 1185.58 & 1151.57 & 0.029 & 0.09 \\
\hline & 3 & & 1390.5 & 1331.45 & 1312.71 & 0.014 & 0.056 \\
\hline & $\bar{x}$ & & 1354.88 & 1293.99 & 1273.17 & 0.017 & 0.061 \\
\hline & $\mathbf{S}$ & & 78.44 & 95.36 & 107.43 & 0.011 & 0.026 \\
\hline & 4 & \multirow{5}{*}{$45^{\circ}$} & 1300.69 & 1235.19 & 1220.04 & 0.012 & 0.062 \\
\hline & 5 & & 1259.51 & 1176.5 & 1146.12 & 0.026 & 0.09 \\
\hline & 6 & & 1354.22 & 1304.54 & 1285.79 & 0.014 & 0.05 \\
\hline & $\overline{\bar{x}}$ & & 1304.81 & 1238.74 & 1217.32 & 0.017 & 0.067 \\
\hline & $\mathbf{S}$ & & 47.49 & 64.09 & 69.87 & 0.007 & 0.02 \\
\hline & 7 & \multirow{5}{*}{$90^{\circ}$} & 1295.86 & 1253.54 & 1244.15 & 0.007 & 0.04 \\
\hline & 8 & & 1283.09 & 1214.55 & 1190.96 & 0.019 & 0.072 \\
\hline & 9 & & 1235.64 & 1147.66 & 1116.25 & 0.027 & 0.097 \\
\hline & $\bar{x}$ & & 1271.53 & 1205.25 & 1183.79 & 0.018 & 0.069 \\
\hline & $\mathbf{S}$ & & 31.73 & 53.55 & 64.25 & 0.01 & 0.028 \\
\hline \multirow{15}{*}{ 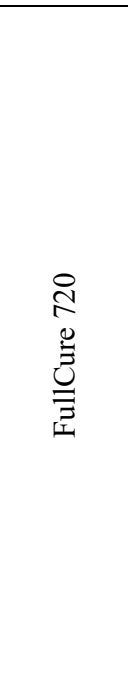 } & 10 & \multirow{5}{*}{$0^{\circ}$} & 1292.17 & 1176.61 & 1133.66 & 0.037 & 0.122 \\
\hline & 11 & & 1331.53 & 1243.96 & 1207.46 & 0.029 & 0.093 \\
\hline & 12 & & 1381.42 & 1308.97 & 1280.88 & 0.021 & 0.073 \\
\hline & $\bar{x}$ & & 1335.04 & 1243.18 & 1207.33 & 0.029 & 0.096 \\
\hline & $\mathbf{S}$ & & 44.73 & 66.18 & 73.61 & 0.007 & 0.025 \\
\hline & 13 & \multirow{5}{*}{$45^{\circ}$} & 1366.08 & 1290.24 & 1250.23 & 0.031 & 0.084 \\
\hline & 14 & & 1302.11 & 1184.88 & 1128.21 & 0.047 & 0.133 \\
\hline & 15 & & 1294.01 & 1167.46 & 1107.35 & 0.051 & 0.144 \\
\hline & $\bar{x}$ & & 1320.73 & 1214.19 & 1161.93 & 0.043 & 0.121 \\
\hline & $\mathbf{S}$ & & 39.48 & 66.43 & 77.17 & 0.011 & 0.032 \\
\hline & 16 & \multirow{5}{*}{$90^{\circ}$} & 1369.50 & 1301.65 & 1277 & 0.019 & 0.068 \\
\hline & 17 & & 1290.62 & 1197.16 & 1162.97 & 0.028 & 0.099 \\
\hline & 18 & & 1374.69 & 1293.72 & 1263.47 & 0.023 & 0.081 \\
\hline & $\overline{\bar{x}}$ & & 1344.94 & 1264.18 & 1234.48 & 0.024 & 0.082 \\
\hline & $\mathbf{S}$ & & 47.11 & 58.17 & 62.29 & 0.005 & 0.016 \\
\hline
\end{tabular}




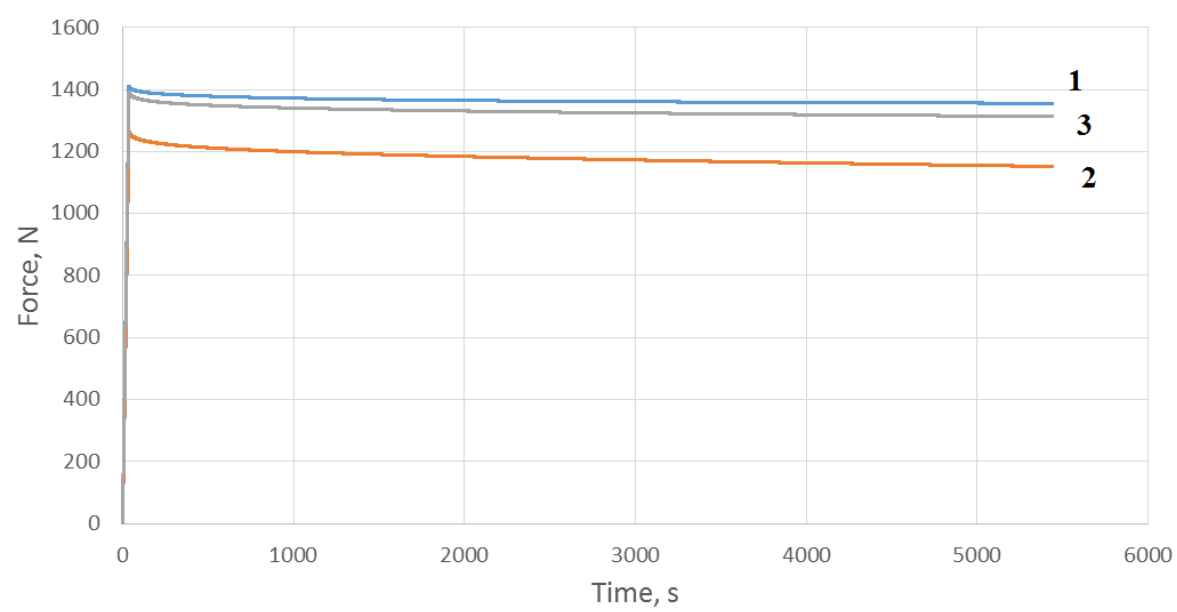

Fig. 3. Exemplary diagram for $P d-0^{\circ}$, VeroWhite

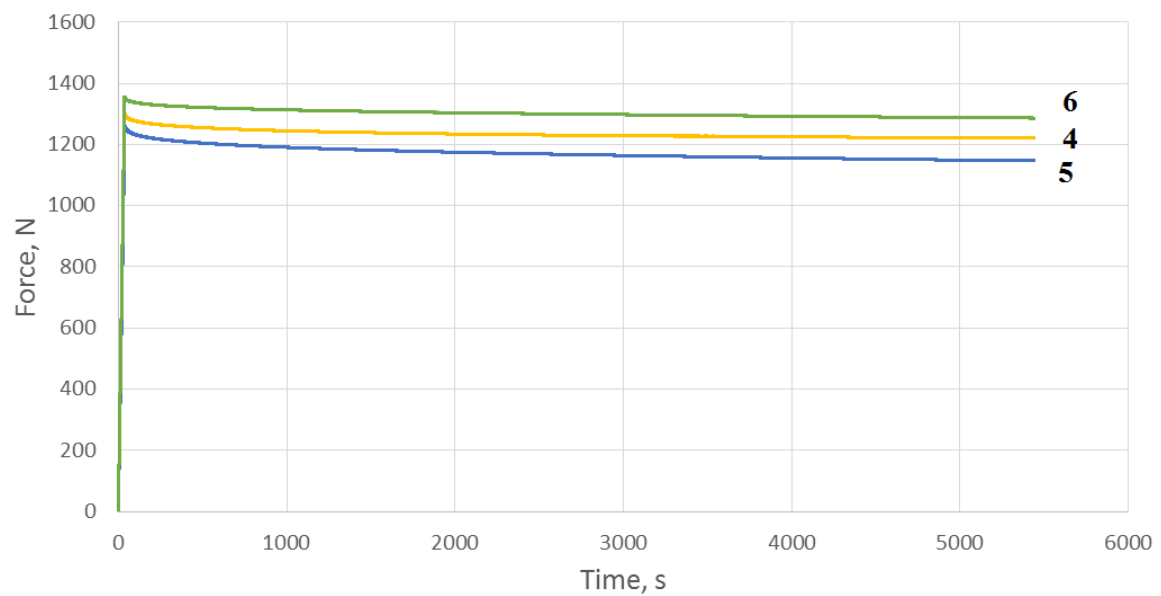

Fig. 4. Exemplary diagram for $P d-45^{\circ}$, VeroWhite

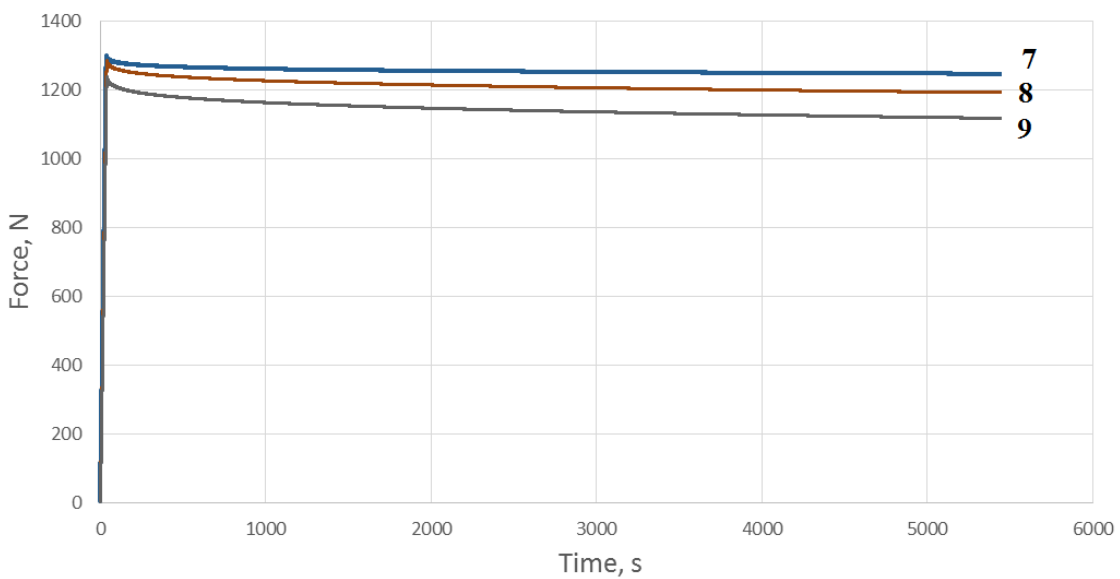


Fig. 5. Exemplary diagram for $P d-90^{\circ}$, VeroWhite

a)
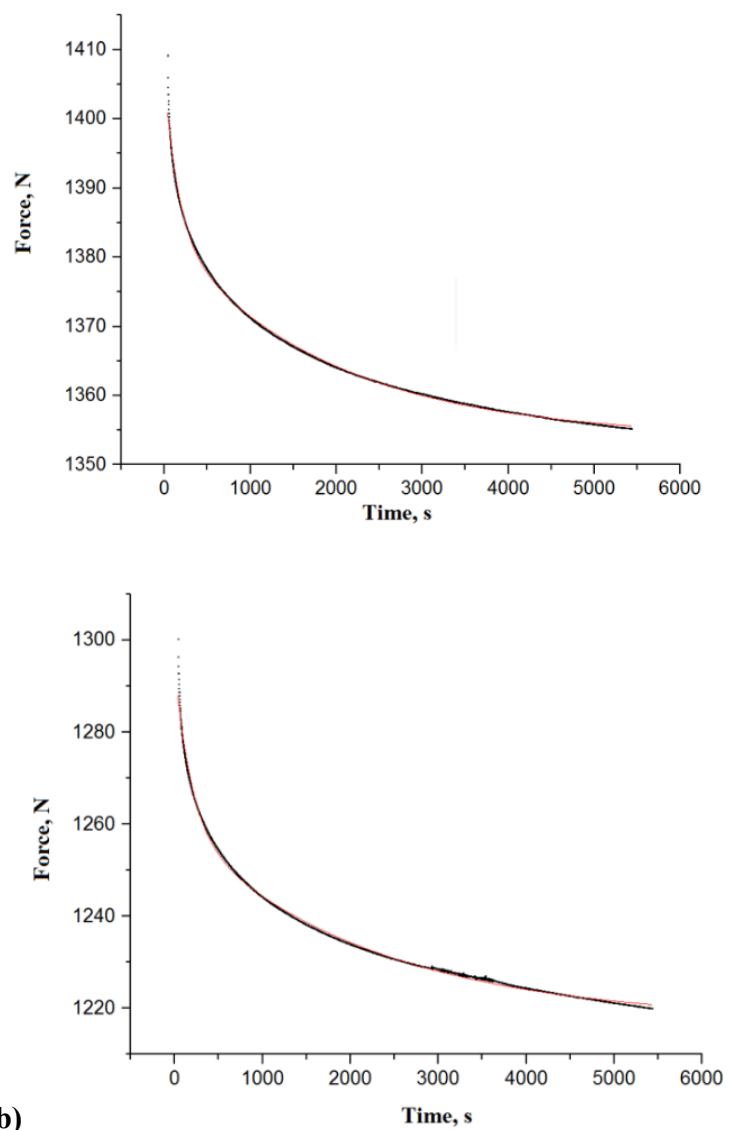

b)

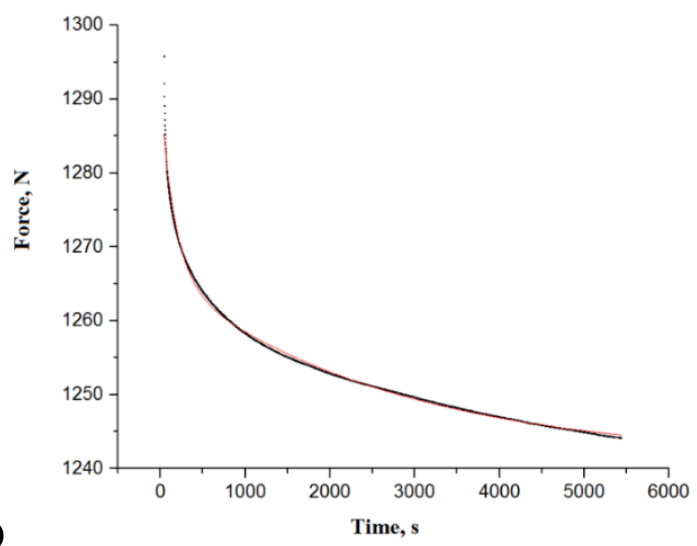

Fig. 6. Figures from Origin software: a) $0^{\circ}$, b) $45^{\circ}$, c) $90^{\circ}$, VeroWhite

Table 2. Exemplary results for above-mentioned diagram 3-5

\begin{tabular}{|c|c|c|c|c|c|c|c|}
\hline$P d^{\circ}$ & $y_{0}$ & $A_{1}$ & $A_{2}$ & $t_{1}$ & $t_{2}$ & $C h i^{2}$ & $R^{2}$ \\
\hline 0 & 1353.945 & 22.709 & 29.57 & 161.706 & 1893.284 & 0.099 & 0.998 \\
\hline 45 & 1217.271 & 35.414 & 42.569 & 184.351 & 2188.143 & 0.333 & 0.998 \\
\hline
\end{tabular}




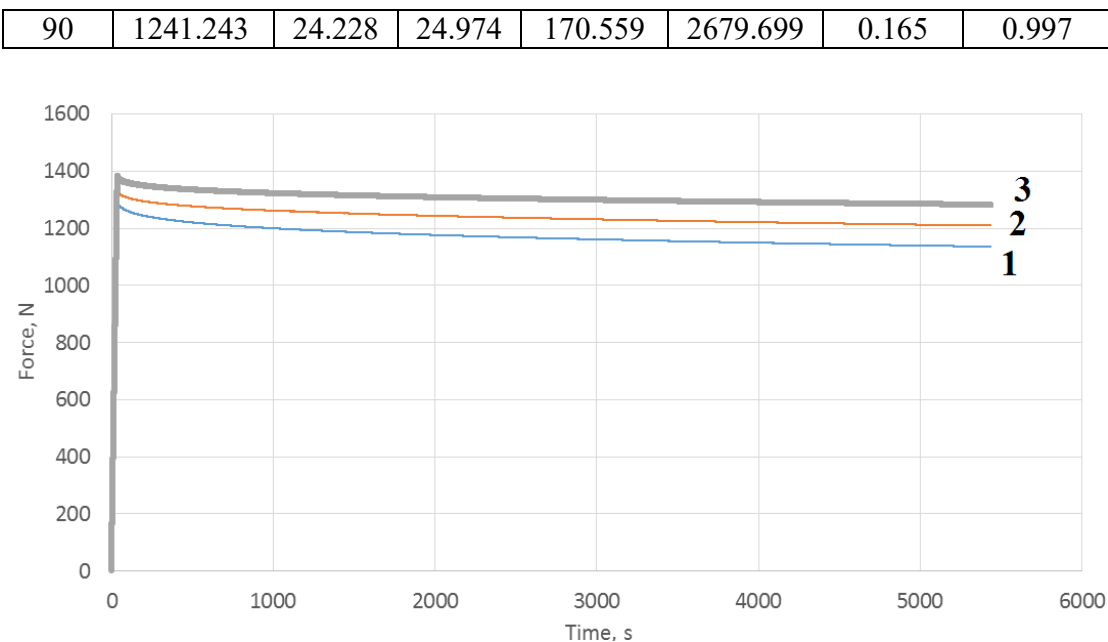

Fig. 7. Exemplary diagram for $P d-0^{\circ}$, FullCure 720

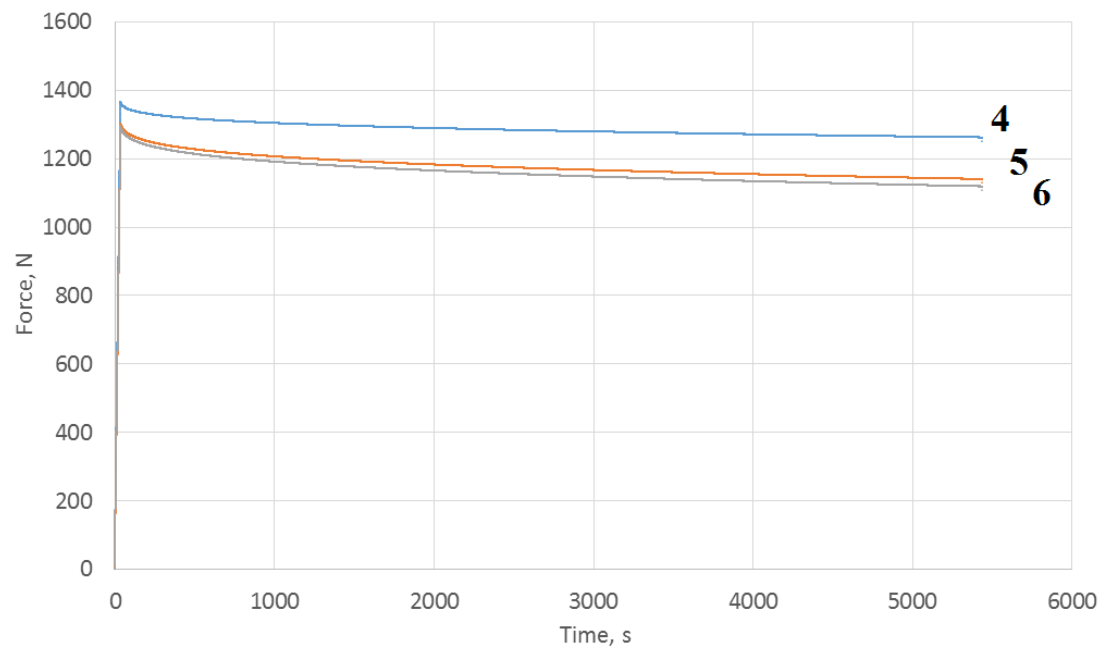

Fig. 8. Exemplary diagram for $P d-45^{\circ}$, FullCure 720 


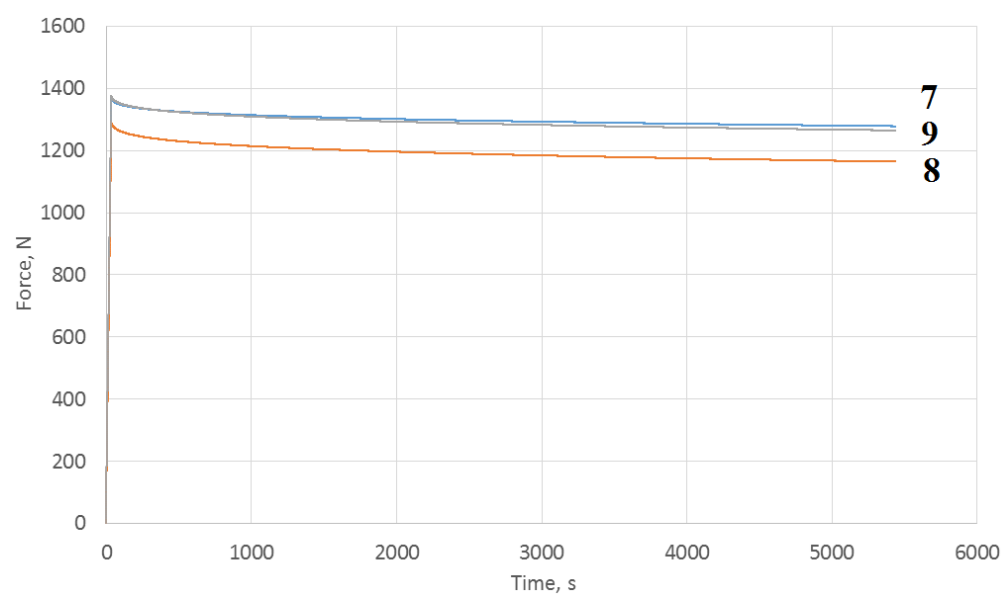

Fig. 9. Exemplary diagram for $P d-90^{\circ}$, FullCure 720

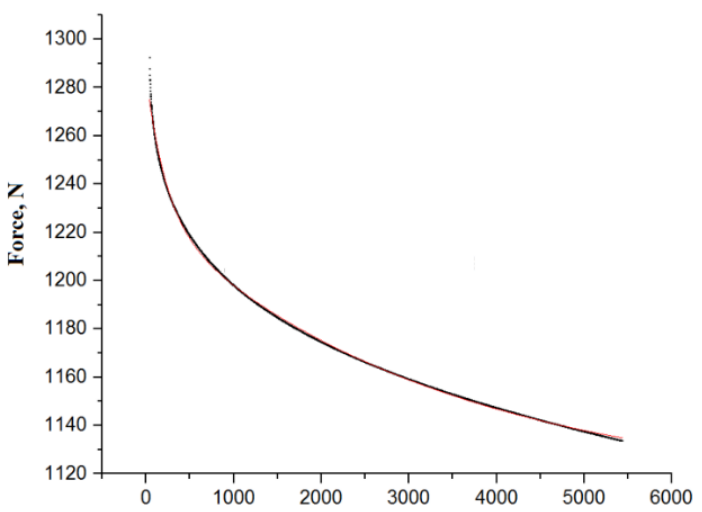

a)

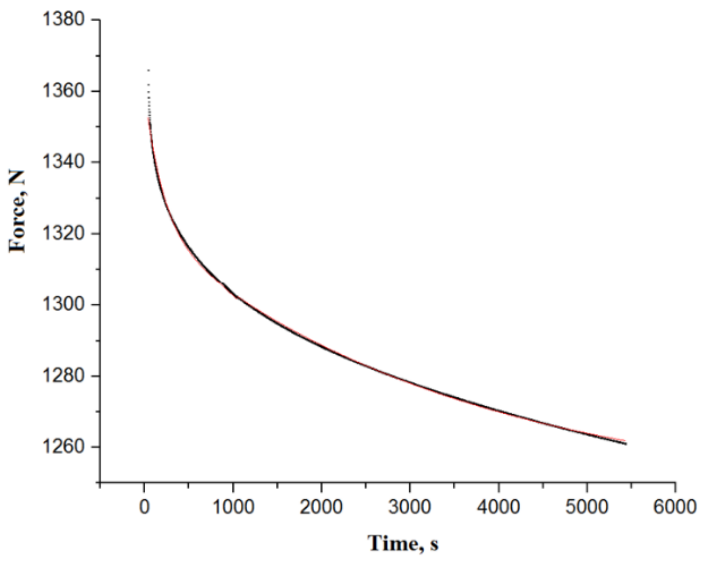




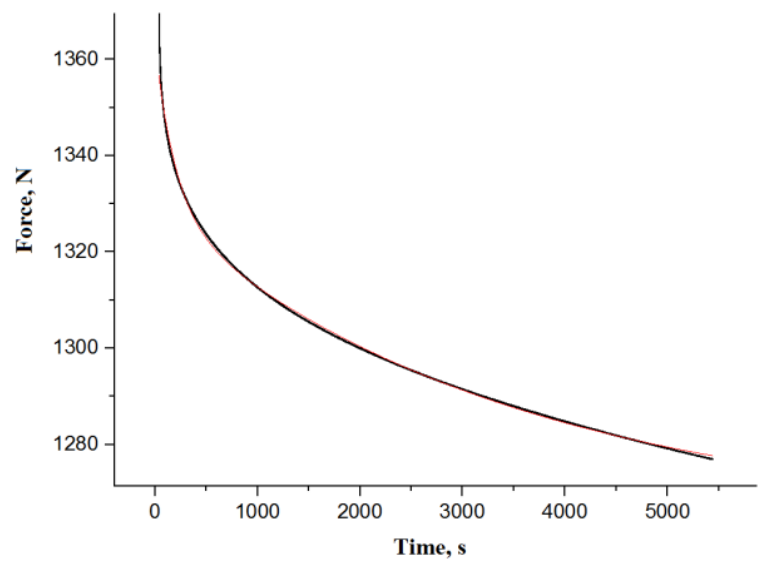

Fig. 10. Figures from Origin software: a) $0^{\circ}$, b) $45^{\circ}$, c) $90^{\circ}$, FullCure 720

Table 3. Exemplary results for above-mentioned diagram 7-9

\begin{tabular}{|c|c|c|c|c|c|c|c|}
\hline$P d^{\circ}$ & $y_{0}$ & $A_{1}$ & $A_{2}$ & $t_{1}$ & $t_{2}$ & $C^{\circ} i^{\wedge} 2$ & $R^{\wedge} 2$ \\
\hline 0 & 1112.911 & 58.247 & 114.422 & 222.382 & 3290.344 & 0.729 & 0.999 \\
\hline 45 & 1245.866 & 37.972 & 76.05 & 207.745 & 3487.365 & 0.348 & 0.999 \\
\hline 90 & 1264.674 & 34.707 & 64.199 & 196.101 & 3407.333 & 0.296 & 0.999 \\
\hline
\end{tabular}

A quantitative analysis of the results of the tests leads to the conclusion that in the case of sample modules made using the PJM technology and using the VeroWhite resin, relaxation of stresses is on a similar level. In all three orientation options $\left(0^{\circ}, 45^{\circ}\right.$, and $\left.90^{\circ}\right)$, the value of relaxation did not exceed 0.07 (respectively $R(t)_{30-90}-0.017,0.017$, and 0.018 , and $R(t)_{c}-$ $0.061,0.067$, and 0.069). In the case of the samples made of the transparent FullCure 720 material, relaxation $\mathrm{R}(\mathrm{t})_{30-90}$, depending on the orientation, was equal to $0.029,0.043$, and 0.024 for the three respective angles of $0^{\circ}, 45^{\circ}$, and $90^{\circ}$. An overall analysis of the test cycle also indicates similar trends for three sample orientation options, i.e.: $0^{\circ}-0.096,45^{\circ}-0.121$, and $90^{\circ}-0.082$. It is clear that the second option, i.e. $45^{\circ}$, is the least advantageous option and demonstrates a nearly two times higher value of stress relaxation.

\section{Conclusion}

Based on the results of the tests of relaxation of stresses in samples made using the PJM technology, the following general conclusions can be drawn.

The five-parameter relaxation function approximates very well the experimental results determined in the course of the compressive stress relaxation. This function may be represents by the general Maxwell model of the rheological properties of the tested materials.

In the case of models made of the VeroWhite material, there is no visible impact of orientation of the models on the building platform on stress relaxation. If the FullCure 720 transparent resin is used to build the models, the value of relaxation for the $45^{\circ}$ angle is nearly two times larger than in the other two cases.

The study was conducted using research facilities purchased with EU funds in the framework of the 2007-2013 Development of Eastern Poland Operational Program, LABIN Project - Support for Innovative Research Facilities of the Kielce University of Technology in Kielce. Priority 1 - Innovative Economy, Measure 1.3 - Support for R\&D Projects. 


\section{References}

1. Cz. Kundera, J. Bochnia, Investigating the stress relaxation of photopolymer O-ring seal models, Rapid Prototyping J. 20, (2014)

2. Cz. Kundera, T. Kozior, Research of the Elastic Properties of Bellows Made in SLS Technology, Adv Mat Res. 874, (2014)

3. Cz. Kundera, T. Kozior, Evaluation of the influence of parameters of FDM technology on the selected mechanical properties of models, Procedia Engineer. 192 (2017)

4. T. Bartkowiak, J.T. Lehner, J. Hyde, Z. Wang, D. Bue Pedersen, H. Norgaard Hansen, C.A. Brown, Multi-Scale areal curvature analysis of fused deposition surfaces, Proceedings - Achieving Precision Tolerances in Additive Manufacturing, (2015).

5. M.C. Leu, N. Guo, Additive manufacturing: technology, applications and research needs. Appl Mech Mater. 8, (2013)

6. Inspekt Mini, Universal Testing Machine Inspekt mini 3kN - User's Guide, Hegewald \& Peschke, Meß- und Prüftechnik GmbH, (2011)

7. PN EN ISO 3384, Rubber, vulcanized or thermoplastic - Determination of stress relaxation in compression at ambient and at elevated temperatures, (2002)

8. J. Skrzypek, Plasticity and creep, (in Polish) PWN, Warsaw, (1986)

9. Cz. Kundera, V. Martsynkowskyy, S. Gudkov, T. Kozior, Effect of rheological parameters of elastomeric ring materials on dynamic of face seals, Procedia Engineer. 17, (2017)

10. ASTM F3049-14, Standard Guide for Characterizing Properties of Metal Powders Used for Additive Manufacturing Processes, ASTM International, West Conshohocken, PA, (2014)

11. Y. Chen, C. Zhezheng, Joint analysis in rapid fabrication of non-assembly mechanisms, Rapid Prototyping J. 17, (2011)

12. S. Fafenrot, N. Grimmelsmann, N. Wortmann, A. Ehrmann, Three-Dimensional (3D) Printing of Polymer-Metal Hybrid Materials by Fused Deposition Modeling, Materials (Basel). 10, (2018)

13. H. Klippstein, H. Hassanin, A.D.D.C Sanchez, Y. Zweiri, L. Seneviratne, Additive Manufacturing of Porous Structures for Unmanned Aerial Vehicles Applications, Adv Eng Mater. 141, (2018)

14. C. Hull, Apparatus for production of three-dimensional objects by stereolithography, US 4575330 A, (1986)

15. C.R. Deckard, J.J. Beaman, J.F. Darrah, Method for selective laser sintering with layerwise cross-scanning, US 5155324 A, (1986)

16. S. Crump, Apparatus and method for creating three-dimensional objects, US 5121329 A, (1989)

17. J.M. Puigoriol-Forcada, A. Alsina, A.G. Salazar-Martín, G. Gomez-Gras, M.A. Pérez, Flexural fatigue properties of polycarbonate fused-deposition modelling specimens, Mater Design. 155, (2018) 